\title{
M-learning e suas Múltiplas Facetas no contexto educacional: Uma Revisão da Literatura
}

Maria das Graças Cleophas

Eduardo Luiz Dias Cavalcanti

Francislê Neri de Souza

Marcelo Brito Carneiro Leão

\begin{abstract}
Resumo
Este artigo apresenta uma investigação sobre o uso dos dispositivos móveis (m-learning) no cenário brasileiro. Para tanto, inicia-se com uma discussão sobre o uso do m-learning no contexto educacional, suscitando algumas questões relevantes sobre essa temática, tais como a sua definição, vantagens e desvantagem sobre a sua integração diante do processo de ensino $e$ aprendizagem das Ciências Naturais. Este trabalho trata-se de uma revisão sistemática da literatura, cuja análise possui teor qualitativo. Foram utilizados como fonte de pesquisa os periódicos da área de educação. Ao todo, analisaramse 32 periódicos, sendo encontrados 36 artigos. Estes artigos foram classificados em seis dimensões que emergiram através da análise realizada. Os resultados deste trabalho apontam para uma preocupação mais latente sobre a produção de plataformas de desenvolvimento em apoio às tecnologias móveis e/ou ferramentas, do que para a compreensão fenomenológica sobre o uso dos dispositivos móveis em sala de aula.
\end{abstract}

Palavras-chave: M-learning; Educação em ciências; Tendências de pesquisa.

\section{Abstract \\ M-learning and its Multiple Facets in the educational context: A Literature Review}

This paper introduces an investigation on the use of mobile (m-learning) devices in the Brazilian scenario. Thereunto, it starts with a discussion on the use of m-learning in the educational context, raising a couple of relevant questions about it, such as its definition, advantages and disadvantage on their integration on the process of teaching and learning of Natural Sciences. This work is a systematic review of the literature with a qualitative approaching analysis. It was used as references several journals from Education. Into 32 journals analyzed were found 36 articles. These articles were classified into six dimensions emerged through this analysis. The results point out to more latent worry about development platforms creation to support mobile technologies and/or tools than to the mobile devices uses on classrooms phenomenological comprehension.

Keywords: M-learning, education, research trends. 


\section{Introdução}

A utilização e a discussão sobre o uso dos dispositivos móveis no contexto educacional brasileiro são relativamente recentes. Os dispositivos móveis estão cada vez mais presentes e sofisticados, e seu potencial para uso na educação é descrito como um "novo paradigma" (LEUNG e CHAN, 2003). O que se sabe é que o m-learning (mobile learning) é considerado um paradigma educativo e está envolto em uma série de possibilidades de aplicações que estão emergindo perante as mudanças necessárias ao processo evolutivo da melhoria da qualidade da educação.

Definir m-learning não é simples e não existe um consenso em relação à sua conceituação entre vários estudiosos (TRAXLER, 2009; CERTAL e CARVALHO, 2011). Para Berge e Muilenburg (2013), ainda não há nenhum conceito definitivo sobre o m-learning. No entanto, algumas definições podem ser retiradas da literatura, tais como a de O'Malley et al. (2003), que consideram qualquer tipo de aprendizagem que acontece quando o aluno não está em um local fixo, predeterminado, ou uma aprendizagem que acontece quando o aluno consegue extrair proveito das inúmeras oportunidades de aprendizagem oferecidas pelas tecnologias móveis.

Para Saccol (2010), o m-learning atua como "processos de aprendizagem apoiados pelo uso de Tecnologias da Informação ou comunicação móveis e sem fio, e que tem como característica fundamental a mobilidade dos aprendizes, que podem estar fisicamente/geograficamente distantes uns dos outros e também de espaços formais de educação, tais como salas de aula, salas de formação, capacitação e treinamento ou local de trabalho" (SACCOL, 2010, p. 25). Moura (2010) define como o processo de aprendizagem que ocorre apoiado pelo uso de dispositivos móveis, tendo como característica fundamental a portabilidade dos dispositivos e a mobilidade dos sujeitos, que podem estar física e geograficamente distantes uns dos outros ou em espaços físicos formais de educação, como a sala de aula. É incontestável, em ambas as definições apresentadas sobre o m-learning, o caráter centrado no aspecto referente à mobilidade do processo, ou seja, o seu surgimento e aplicação no contexto escolar, ou fora dele, uma vez que surgiu para "modernizar" o processo formal, não formal e informal de ensino.

Dado o exposto, percebe-se que a aprendizagem móvel é capaz de fornecer um mecanismo útil para enriquecer a aprendizagem dos alunos (OKITA et al., 2013). O rápido desenvolvimento para a implantação de tecnologia móvel no âmbito escolar oferece aos alunos novas oportunidades para aumentar o engajamento, a motivação e a aprendizagem (LIN et al., 2012 apud AHMED e PARSONS, 2013). O que se pretende com o uso dos dispositivos móveis no contexto educacional é educar os indivíduos não apenas para pesquisar e utilizar uma variedade de informações, mas também para criar novos elementos valiosos que possam ser usados para solução de problemas que estejam ligados à vida social desses indivíduos (JAMEE e WONGYU, 2013), e, nesta perspectiva, o uso desse dispositivo tem muito a ser explorado. Como o mlearning está se desenvolvendo rapidamente, ramificações sobre a sua usabilidade estão sendo 
criadas (BERGE e MUILENBURG, 2013), e espera-se que sejam desenvolvidas estratégias que contemplem mais este tema, e que, sobretudo, seu uso seja mais difundido no meio educacional, por diferentes áreas de conhecimento.

Traxler (2011) descreveu cinco maneiras pelas quais o m-learning oferece novas oportunidades para a aprendizagem: (1) aprendizagem contingente, permitindo que os alunos possam responder e reagir às experiências vivenciadas em diferentes contextos; (2) a aprendizagem situada, que ocorre em ambientes aplicáveis à aprendizagem; (3) a aprendizagem autêntica, com as tarefas diretamente relacionadas aos objetivos de aprendizagem que se deseja atingir; (4) a aprendizagem consciente do contexto, em que a aprendizagem é informada pela história e pelos objetivos; e (5) a aprendizagem personalizada, ou seja, específica para cada aluno, em termos de habilidades, interesses e preferências. E podemos acrescentar a Aprendizagem Ativa, que está relacionada à responsabilização pela própria aprendizagem, desenvolvimento com competência de alto nível cognitivo num processo centrado no aluno e para o aluno (NERI de SOUZA, 2006). Mediante as possibilidades apresentadas pelo m-learning, é possível perceber que inúmeras estratégias didáticas podem ser utilizadas por professores e alunos, visando desta forma fornecer uma nova maneira de dinamizar o ensino tradicionalista, além de favorecer a inclusão tecnológica de maneira proveitosa para fins didático-pedagógicos. Desta forma, sabemos que as tecnologias móveis estão revolucionando a educação, ao transformar a sala de aula convencional, ao inserir aplicações interativas que têm o potencial de melhorar as experiências de aprendizagem dos alunos (SCORNAVACCA, HUFF e MARSHALL, 2009). Vale frisar que a implantação das tecnologias móveis no contexto escolar tende a favorecer não apenas contribuições perante a melhoria do aprendizado dos alunos, mas também pela capacidade inovadora diante da práxis docente. Uma comparação entre o m-learning e o ensino tradicional pode ser vista na Tabela 1.

Tabela 1 - Comparação entre o ensino tradicional (quando fornecido em sala de aula) e o M-learning (Adaptado de Berge e

Muilenburg, 2013).

\begin{tabular}{|c|c|c|}
\hline & Ensino Tradicional & M-learning \\
\hline Tempo & $\begin{array}{l}\text { Muitas vezes limitado por } \\
\text { horários escolares formais. }\end{array}$ & $\begin{array}{l}\text { Sem restrições de tempo (o aluno pode } \\
\text { estudar em qualquer lugar, e a qualquer } \\
\text { hora, basta ter o dispositivo móvel). }\end{array}$ \\
\hline Personalização & $\begin{array}{c}\text { Limitado sob todos os } \\
\text { aspectos de diferenciação e } \\
\text { conceitos ensinados. }\end{array}$ & $\begin{array}{l}\text { Personalizado através de aplicações, } \\
\text { revisões, conceitos etc. }\end{array}$ \\
\hline $\begin{array}{c}\text { Ensino } \\
\text { individualizado }\end{array}$ & Não individual (coletivo). & $\begin{array}{c}\text { Individual, ou seja, pode ser altamente } \\
\text { particular. }\end{array}$ \\
\hline Contexto & $\begin{array}{l}\text { Limitado a um local } \\
\text { geograficamente definido. }\end{array}$ & $\begin{array}{l}\text { A aprendizagem pode ocorrer em várias } \\
\text { situações distintas, e em ambientes } \\
\text { socialmente diversos. }\end{array}$ \\
\hline
\end{tabular}




\begin{tabular}{|c|c|c|}
\hline $\begin{array}{c}\text { Formal/Não } \\
\text { formal/informal }\end{array}$ & $\begin{array}{l}\text { Formal - centrado na sala de } \\
\text { aula. }\end{array}$ & $\begin{array}{l}\text { Pode ser aplicado no ensino formal, não } \\
\text { formal e também informal. }\end{array}$ \\
\hline $\begin{array}{l}\text { Aspecto sócio- } \\
\text { conectividade }\end{array}$ & $\begin{array}{l}\text { As conexões são predefinidas } \\
\text { e habilitadas para este tipo de } \\
\text { contexto. }\end{array}$ & $\begin{array}{l}\text { Não é preciso ter conexão ativa durante } \\
\text { o aprendizado, basta possuir as } \\
\text { atividades ou tarefas, e estas podem ser } \\
\text { obtidas por mensagens ou quando se } \\
\text { tiver acesso a uma rede de conexão } \\
\text { qualquer. }\end{array}$ \\
\hline Espontaneidade & Não é espontâneo. & Altamente espontâneo. \\
\hline $\begin{array}{l}\text { Direção e } \\
\text { sentido da } \\
\text { interação }\end{array}$ & $\begin{array}{l}\text { Geralmente do professor para } \\
\text { o aluno (unilateral). }\end{array}$ & $\begin{array}{l}\text { Possiblidades de ser dos alunos para o } \\
\text { professor e para os colegas que } \\
\text { participam do seu processo de } \\
\text { aprendizagem (bilateral). }\end{array}$ \\
\hline Colaborativa & $\begin{array}{l}\text { O ensino tradicional tende a } \\
\text { não favorecer de forma } \\
\text { acentuada aspectos } \\
\text { colaborativos entre os sujeitos } \\
\text { envolvidos no processo de } \\
\text { ensino e aprendizagem. }\end{array}$ & $\begin{array}{l}\text { Diversas estratégias didáticas podem } \\
\text { ser fomentadas, colaborativamente, } \\
\text { entre os alunos. }\end{array}$ \\
\hline $\begin{array}{l}\text { Capacidade de } \\
\text { descobrir } \\
\text { situações que } \\
\text { possam } \\
\text { favorecer o } \\
\text { ensino e a } \\
\text { aprendizagem }\end{array}$ & $\begin{array}{l}\text { As descobertas realizadas } \\
\text { pelos alunos são quase } \\
\text { sempre centradas nas } \\
\text { informações do professor. }\end{array}$ & $\begin{array}{l}\text { Os alunos podem descobrir novos } \\
\text { aplicativos ou tarefas que facilitem o } \\
\text { processo de ensino e aprendizagem, } \\
\text { além de compartilhá-los com os demais } \\
\text { colegas e professor. }\end{array}$ \\
\hline Avaliação & $\begin{array}{l}\text { Os alunos têm o seu } \\
\text { conhecimento mensurado a } \\
\text { partir dos conteúdos } \\
\text { fornecidos pelo professor, o } \\
\text { qual elabora uma prova e } \\
\text { qualifica o aluno através do } \\
\text { seu desempenho. }\end{array}$ & $\begin{array}{l}\text { Diferentes métodos avaliativos podem } \\
\text { ser disponibilizados aos alunos (desde } \\
\text { que sejam minuciosamente planejados). }\end{array}$ \\
\hline
\end{tabular}

Conforme salientam Wong e Looi (2011) em suas pesquisas, o m-learning pode contemplar diferentes aspectos de aprendizagem: formal e informal, personalizado e social, ambiente "físico" e virtual. Ele pode melhorar a aprendizagem ao longo do tempo e os locais, permitindo acesso ubíquo aos alunos e professores, além de acesso a fontes de informação. Para Cinque (2009), o uso dos dispositivos móveis oferece várias vantagens sob o ponto de vista dos professores, tais como: "estar além da sala de aula"; rápido acesso à informação; anotações gráficas e multimídicas; respostas em tempo real; acessibilidade e colaboração.

Todavia, mesmo com toda a falácia que tem sido gerada acerca do uso do m-learning, ainda são considerados incipientes os estudos em relação aos benefícios e malefícios destes dispositivos perante a sua aplicabilidade na educação, além de estratégias que possam favorecer o seu uso. Seus fundamentos teóricos ainda não amadureceram (MUYINDA, 2007). Os

DOI: Em andamento. 
pressupostos teóricos são compostos de várias vertentes, de modo que não se pode atribuir a uma única teoria de aprendizagem a responsabilidade de alicerçar todos os seus princípios educativos. Apesar do alto grau de inserção dos dispositivos móveis na sociedade atual, a simples disponibilidade dessa tecnologia por si só não garante que o seu potencial será utilizado em termos de aprendizagem, nem aceito de forma homogênea por todos (FERREIRA et al., 2012). Isso resulta na percepção de que, até agora, o m-learning ainda não tem causado grande impacto no contexto educacional (LIU, HAN e LI, 2010).

Jonassen, Carr e Yueh (1998, p. 1) sinalizaram que "as tecnologias móveis", tais como telefones celulares, smartphones, Ipads, tablets, computadores portáteis, etc., não devem ser consideradas instrumentos recursivos que promovem o aprendizado, e sim ferramentas que podem ser usadas como aporte para a construção do conhecimento, ou seja, os alunos podem aprender com o seu uso, e não a partir deles. Mas é fundamental compreender que, para esses dispositivos atuarem como ferramentas no contexto educacional, se faz necessário traçar e aplicar várias estratégias metodológicas que viabilizem o seu uso perante o aprendizado de conteúdos diversos. Não obstante, durante o caminho percorrido para a integração dos dispositivos móveis em sala de aula, é encontrada resistência das famílias e dos professores, pois os mesmos consideram esses dispositivos mais como meios de comunicação e entretenimento do que possíveis ferramentas educativas (GRUND e GIL, 2011).

Neste manuscrito, tivemos como objetivo identificar e analisar, por meio de uma revisão sistemática da literatura, as principais tendências em relação às pesquisas que abarcaram diferentes aspectos relacionados ao m-learning, como ferramentas de suporte, aplicabilidade, desenvolvimento de plataformas, etc. $O$ que pretendemos é traçar um perfil sobre a divulgação do m-learning, ou seja, verificar como tem sido o andamento das pesquisas em relação a esta temática. Dessa forma, seremos capazes de mapear estas pesquisas, através da varredura nos periódicos selecionados, além de mostrarmos as suas características em relação às tendências de pesquisa.

Para atingir tais objetivos, trabalhos sobre a temática foram pesquisados em artigos completos publicados em periódicos da área de educação (ensino de ciências e/ou matemática) ou com foco no uso das Tecnologias da Informação e Comunicação (TIC) (Tabela 2). As diversas considerações metodológicas acerca dos critérios de seleção dos artigos nestas fontes de pesquisa são apresentadas a seguir. Cabe destacar que o levantamento realizado não pretendeu ser fatigante e, dadas a qualidade e a variedade em relação às revistas analisadas, acredita-se que os dados levantados apontam as principais tendências em relação à temática investigada e como ela está permeada diante do contexto educacional. 


\section{Aspectos metodológicos adotados}

Neste artigo, foram investigadas as características apresentadas em relação à usabilidade e aplicabilidade da temática em questão, em periódicos voltados para a área de Ensino. Como meta, visou-se fazer uma varredura em diversas revistas encontradas na base Scielo, no Google Acadêmico e nos próprios sítios destas revistas. Buscou-se mapear como a temática desta pesquisa estava sendo disseminada, além de verificar a aceitabilidade ou inserção deste tema nas mesmas.

Segundo Kitchenham (2004), uma revisão sistemática da literatura é um método de avaliação e interpretação investigativas relevante, realizado para uma determinada questão de investigação, uma determinada área de interesse ou um evento. Ao todo, foram analisadas 32 revistas utilizadas para a divulgação de artigos na área de educação, ensino de ciências e TIC. Tais revistas apresentaram diferentes conceitos em relação a sua qualidade, que é mensurada e indicada pelo Qualis da Capes (o Qualis de cada revista é encontrado no site: www.qualis.capes.gov.br; para ter acesso a ele, basta conhecer o nome da revista ou o seu ISBN). A lista contendo as revistas que foram investigadas e o número de artigos encontrados está disponível na Tabela 2:

Tabela 2: Revistas consultadas no Portal de Periódico Capes.

\begin{tabular}{|c|c|}
\hline REVISTAS & $\begin{array}{lcc}\text { № } & \text { de } & \text { Artigos } \\
\text { encontrados } & \end{array}$ \\
\hline 1. Alexandria: Revista de Educação em Ciência e Tecnologia & 0 \\
\hline 2. Caderno Brasileiro de Ensino de Física & 0 \\
\hline 3. Cadernos CEDES & 0 \\
\hline 4. Ciência e Educação & 0 \\
\hline 5. Ciência e Ensino & 0 \\
\hline 6. Educação e Pesquisa & 0 \\
\hline 7. Educação e Realidade & 0 \\
\hline 8. Educação e Sociedade & 0 \\
\hline 9. Ensaio: Pesquisa em Educação em Ciências & 0 \\
\hline 10. Experiências em Ensino de Ciências & 0 \\
\hline 11. Investigações em Ensino de Ciências & 0 \\
\hline 12. Revista Brasileira de Informática na Educação & 6 \\
\hline 13. Zetetike (Faculdade de Educação da UNICAMP) & 0 \\
\hline 14. Educação, Formação \& Tecnologias & 4 \\
\hline 15. TECCOGS: Revista Digital de Tecnologias Cognitivas & 0 \\
\hline 16. RENOTE. Revista Novas Tecnologias na Educação & 15 \\
\hline 17. Revista Latinoamericana de Tecnología Educativa & 0 \\
\hline 18. Tecnologia Educacional & 0 \\
\hline 19. CTS. Ciencia, Tecnología y Sociedad & 0 \\
\hline 20. Revista Brasileira de Ensino de Ciência e Tecnologia & 0 \\
\hline 21. Revista de la Facultad de Ciencia y Tecnologia & 0 \\
\hline 22. Educação \& Tecnologia & 0 \\
\hline 23. JISTEM Revista de Gestão da Tecnologia e Sistemas de Informação & 1 \\
\hline
\end{tabular}

DOI: Em andamento. 


\begin{tabular}{|l|l|}
\hline 24. Ciência e Tecnologia & 0 \\
\hline 25. Revista Tecnologia e Sociedade (Online) & 0 \\
\hline 26. Tecnologia \& Cultura (CEFET/RJ) & 0 \\
\hline 27. Acta Scientiarum Education & 1 \\
\hline 28. Informação \& Informação & 0 \\
\hline 29. ETD. Educação Temática Digital & 0 \\
\hline 30. Educação online & 3 \\
\hline 31. Informática na Educação: Teoria e Prática & 5 \\
\hline 32. Udesc Virtual - Online & 1 \\
\hline
\end{tabular}

Como o tema em questão é relativamente novo, foram analisadas as publicações de cada periódico no período de 2007 a junho de 2013. Todos os periódicos pesquisados estavam disponíveis na internet no banco de dados da Scielo, no Google Acadêmico ou nos sites das revistas. Das 32 revistas analisadas, foi encontrado um total de 36 artigos contendo alguma palavra-chave que direcionava ao tema m-learning. Os termos utilizados, dentro de um espectro de palavras-chave, para selecionar os artigos foram: m-learning; mobile; celular; tablets; dispositivos móveis; ubíquo. Após a etapa de seleção dos artigos, estes foram analisados minuciosamente, visando posteriormente à criação de dimensões para classificá-los de acordo com a sua finalidade de aplicação ou tipo de abordagem.

Esta pesquisa se apoia nos pressupostos da análise documental. Segundo Caulley (1981 apud LÜDKE e ANDRÉ, 2013), este tipo de análise busca identificar informações factuais nos documentos a partir de questões ou hipóteses de interesse. Para a aplicação da metodologia supracitada, foram necessárias as seguintes etapas que fundamentaram o seu escopo: 1) préanálise, fase de organização do material disponível; 2) exploração do material, que consistiu na codificação dos dados brutos e, consequentemente, na agregação em categorias; e 3) o tratamento dos resultados, a inferência e a interpretação.

\section{Resultados obtidos}

Os resultados aqui apresentados serão divididos em etapas, visando a uma melhor sistematização da análise dos dados, bem como à discussão destes.

\section{Criação das dimensões de análise categorial}

As dimensões categoriais foram criadas de acordo com a conjuntura apresentada por cada artigo analisado, ou seja, emergiram da leitura dos dados. Para a concretização desta etapa, foram necessárias a leitura dos artigos e, em seguida, a separação destes por semelhança apresentada em relação a sua abordagem. Foram elaboradas 6 (seis) dimensões distintas, conforme apresentadas abaixo:

Tabela 3: Número de artigos alojados em cada dimensão. 


\begin{tabular}{|c|l|c|c|}
\cline { 2 - 4 } \multicolumn{1}{c|}{} & \multicolumn{1}{|c|}{ DIMENSÕES CATEGORIAIS } & $\begin{array}{c}\text { QUANT. DE } \\
\text { ARTIGOS }\end{array}$ & $\begin{array}{c}\text { VALORES } \\
\text { PERCENTUAIS (\%) }\end{array}$ \\
\hline a) & $\begin{array}{l}\text { Discussão sobre o m-learning } \\
\text { (conceito, teorias, estratégias de } \\
\text { uso) }\end{array}$ & 7 & 19,44 \\
\hline b) & $\begin{array}{l}\text { Plataformas de desenvolvimento em } \\
\text { apoio tecnologias } \\
\text { móveis/ferramentas }\end{array}$ & 10 & 27,78 \\
\hline c) & $\begin{array}{l}\text { Usabilidade do celular no contexto } \\
\text { escolar }\end{array}$ & 5 & 13,88 \\
\hline d) & $\begin{array}{l}\text { Inclusão de dispositivos portáteis na } \\
\text { sala de aula }\end{array}$ & 3 & 8,34 \\
\hline e) & $\begin{array}{l}\text { Objetos de Aprendizagem para } \\
\text { execução em dispositivos móveis }\end{array}$ & 5 & 13,88 \\
\hline f) & $\begin{array}{l}\text { Mobile Learning Engine Moodle } \\
\text { (MLE-Moodle) }\end{array}$ & 6 & 16,67 \\
\hline
\end{tabular}

\section{Breve descrição sobre cada dimensão}

\section{a) Discussão sobre o m-learning (conceito, teorias, estratégias de uso)}

Segundo Traxler (2009), o m-learning ainda consiste em um campo imaturo, tanto em termos tecnológicos quanto pedagógicos, porém a discussão sobre ele pode trazer contribuições à medida que avançam as pesquisas na área. $O$ conceito de $\mathrm{m}$-learning no contexto nacional ainda é bastante controverso. De acordo com Ledesma (2013), o conceito de m-learning pode ser traduzido para o Português por aprendizagem móvel, ou entendido como integração das tecnologias móveis em contexto educativo. Apesar da diversidade de conceitos, uma característica comum emerge das concepções de vários autores, o que de fato é um atributo latente do m-learning: a aprendizagem a todo e qualquer momento com tecnologias sem fio (WANG, 2004; SHARPLES, 2006; TRAXLER, 2007; TWISS, 2008).

\section{b) Plataformas de desenvolvimento em apoio às tecnologias móveis/ferramentas}

Esta dimensão foi elaborada mediante os inúmeros artigos encontrados a partir desta análise, cujo teor estava relacionado ao uso de plataformas de desenvolvimento, tendo como meta apoiar as tecnologias móveis. Tais plataformas desempenham um papel importante diante da popularização do uso do m-learning para o processo de ensino e aprendizagem. Muitas destas plataformas são gratuitas e, sem elas, é impossível o desenvolvimento de materiais ou aplicativos que possam ser suportados por dispositivos móveis. É importante denotar que, para construir

DOI: Em andamento. 
materiais didáticos, ou não, para estes dispositivos, é necessário conhecer linguagem de programação. Ou seja, a linguagem destas plataformas é mutável de acordo com o sistema operacional, o que significa que para o iOS a plataforma (Objective) é diferente da utilizada pelo sistema android (geralmente, Java).

\section{c) Usabilidade do celular no contexto escolar/estratégias de uso}

Os dispositivos móveis podem ser usados para aceder à informação em qualquer lugar e a qualquer hora, por isso é adequada a integração destes dispositivos em atividades de aprendizagem que vão ao encontro de suas características (MOURA, 2010). Os dispositivos móveis, ao acompanharem os utilizadores nas suas experiências cotidianas, tornam-se uma fonte conveniente de informação e meio de comunicação que auxilia a aprendizagem (CORBEIL e VALDES-CORBEIL, 2007). Desta forma, por exemplo, um professor pode propor estratégias para revisar o conteúdo, enviando sms para seus alunos. Estamos caminhando para uma era onde será inconcebível a ideia de não termos à disposição os dispositivos móveis no contexto educacional. Esta dimensão teve o objetivo de verificar como os artigos descrevem as diversas formas de emprego dos dispositivos móveis no ambiente escolar.

\section{d) Inclusão de dispositivos portáteis na sala de aula}

Esta dimensão descreve os artigos que relatam diferentes possibilidades de inserir os dispositivos móveis em sala de aula, mostrando que a criatividade do professor pode favorecer o seu processo de implantação. Incluir os dispositivos em sala de aula implica numa série de situações distintas. A primeira delas esbarra nas políticas que regem a inserção destes dispositivos no contexto educacional. O Brasil ainda não possui uma política que possa reger este processo de inserção de forma correta, de modo a dar diretrizes de como executar esta inserção sem polêmica nas salas de aula. Segundo Plesch et al. (2013), a aprendizagem móvel é uma das principais tendências para o cenário educacional. Contudo, é necessário pensar no contexto social em que o aluno está inserido, pois muitas dessas tecnologias não estão disponíveis. Prensky (2001) e vários outros autores têm procurado descrever as mudanças nos alunos em termos de diferenças geracionais, medindo essas diferenças pela facilidade com que se adaptam às novas tecnologias.

\section{e) Objetos de Aprendizagem para execução em dispositivos móveis}

Formulou-se esta dimensão através da incidência de artigos que relataram o uso destes objetos de aprendizagem apoiados em dispositivos móveis. Não existe ao certo uma definição universal que possa conceituar o que seriam "objetos de aprendizagem", como, por exemplo, animações, simulações, imagens, fragmentos de vídeo, fragmentos de áudio, textos, gráficos, jogos, etc. No entanto, eles podem ser considerados como recursos que possam dar aporte ao aprendizado. Sosteric e Hosemeier (2002) definiram como sendo arquivos digitais (imagens, filmes, etc.) que podem ser utilizados com fins educacionais e que incluem, internamente ou através de ligações, sugestões sobre o contexto apropriado de uso. Para Wiley (2002), objetos de 
aprendizagem são "quaisquer recursos digitais que possam ser reutilizados para o suporte ao ensino". Já Audino e Nascimento (2010) entendem que os objetos de aprendizagem são qualquer material digital, como textos, animações, vídeos, páginas web, usados, de forma isolada ou em combinação, com fins educacionais. Para os autores, trata-se de um instrumento autônomo, que pode ser utilizado como módulo de determinado conteúdo, bem como de um conteúdo completo, podendo ainda ser incorporado a múltiplos aplicativos.

\section{f) Mobile Learning Engine Moodle (MLE-Moodle)}

Esta dimensão visa verificar os artigos que versaram sobre o uso das plataformas de aprendizagem à distância Moodle (MLE-MOODLE, 2009). O Mobile Learning Engine Moodle é uma interface que tem um aplicativo adaptado e que pode ser utilizado por dispositivos móveis. De acordo com Ribeiro e Medina (2009), o MLE-Moodle (Mobile Learning Engine Moodle) é de código-fonte livre, totalmente gratuito e personalizável, vinculado ao Ambiente Virtual de Aprendizagem (AVA) Moodle. Segundo Yinling (2006 apud Ribeiro e Medina, 2009), com a criação do projeto MLE-Moodle, tornou-se possível acessar o conteúdo criado diretamente no Moodle, utilizando-se dispositivos móveis. Qualquer alteração efetuada no Moodle convencional é, automaticamente, convertida para o MLE-Moodle. Assim, garante-se a mobilidade do aprendiz, que poderá ter acesso ao conteúdo do Moodle em qualquer lugar e hora, bastando apenas querer. Assim, o objetivo do projeto Mobile Learning Engine Moodle é garantir que, fora do ambiente da escola, ou de sua casa (onde geralmente se utiliza o Moodle tradicional instalado em seu computador ou notebook), o aprendiz possa continuar a ter acesso ao seu material.

\section{DIMENSÃO versUs REVISTA}

Com o intuito de organizar os artigos selecionados e enquadrá-los de acordo com as seis dimensões elaboradas, foi criada uma Tabela (Anexo 1) que correlaciona o artigo com a sua respectiva dimensão, a revista na qual foi publicado, título do artigo, além dos dados sobre a sua divulgação (volume, número e ano). O objetivo de tal Tabela (Anexo 1) é divulgar o andamento das pesquisas que envolvem a temática m-learning no contexto da educação em Ciências.

Ao analisarmos as revistas que foram as fontes de nossa pesquisa, percebemos, dentro deste universo investigado, que ainda não são amplamente divulgados artigos que versem sobre a temática m-learning no contexto da educação em Ciências. As pesquisas ainda são carentes em termos de aplicação, desenvolvimento de objetos de aprendizagem, discussão sobre o processo de inclusão de dispositivos móveis em sala de aula, etc., para o ensino das Ciências. Ainda temos um longo caminho a percorrer. Países como Egito, China e Índia estão avançando rapidamente nas pesquisas sobre o m-learning, além de utilizarem os dispositivos móveis com maior naturalidade no contexto escolar. No início de 2013, a UNESCO (Organização das Nações Unidas para a Educação, a Ciência e a Cultura) publicou um guia recomendando o uso dos dispositivos móveis na educação. Esta, provavelmente, foi uma forma de provocar os governos a inserirem 
políticas públicas educacionais que valorizem a inserção de celulares ou outros dispositivos móveis como um recurso didático nas salas de aula. Neste guia, foram apresentados treze bons motivos que favorecem o uso do celular em sala de aula, tais como: amplia o alcance e a equidade em educação; melhora a educação em áreas de conflito ou que sofreram desastres naturais; assiste alunos com deficiência; otimiza o tempo na sala de aula; permite que se aprenda em qualquer hora e lugar; constrói novas comunidades de aprendizado; é capaz de dar suporte à aprendizagem in loco; aproxima o aprendizado formal do informal; provê avaliação e feedback imediatos; facilita o aprendizado personalizado; causa melhoria na aprendizagem contínua; melhora a comunicação; e maximiza a relação custo-benefício da educação.

\section{DADOS QUANTITATIVOS versUs DIMENSÃO}

O Gráfico 1, abaixo, ilustra a incidência do número de artigos classificados e atribuídos a cada dimensão. Tal gráfico mostra que a maioria das pesquisas divulgadas nas revistas analisadas se concentra na criação de plataformas de desenvolvimento de tecnologias móveis/ferramentas. Em seguida, vêm as discussões sobre as teorias, conceitos, estratégias de uso sobre o m-learning. Trabalhos que envolveram a plataforma Moodle para celular representaram $16,67 \%$ da preferência em termos de pesquisas. A usabilidade dos dispositivos móveis no contexto escolar representa apenas $13,89 \%$, empatando com a categoria Objetos de Aprendizagem para execução em dispositivos móveis. Foi praticamente irrelevante o número de trabalhos que apresentaram estratégias de uso voltadas para o ensino das Ciências (Química, Física e Biologia); já para a área de Matemática, foram encontrados alguns trabalhos, mostrando, assim, que esta área, possivelmente, apresenta um maior interesse dos pesquisadores em atrelar o uso do m-learning à matemática. Em último lugar, emergiu a dimensão sobre a inclusão dos dispositivos portáteis em sala de aula. Este dado corrobora o que temos presenciado dentro do cenário brasileiro, pois é notório que o uso dos dispositivos móveis no contexto educacional, mais precisamente na sala de aula, está envolto em uma série de paradigmas. Muitas escolas no Brasil proíbem de forma veemente que os alunos utilizem o celular em sala; outras, através de programas educacionais promovidos pelos governos, disponibilizam tablets para os professores das escolas públicas. Esta divergência de opiniões quanto ao uso dos dispositivos móveis em sala de aula está centrada, em grande parte, na falta de formação continuada para os professores, pois, se estes não estão aptos a integrarem os dispositivos em suas atividades docentes, não serão favoráveis a que os alunos utilizem tais dispositivos em suas salas de aula. Kenski (2009) afirma que:

"Um dos grandes desafios que os professores brasileiros enfrentam está na necessidade de saber lidar pedagogicamente com alunos e situações extremas: dos alunos que já possuem conhecimentos avançados e acesso pleno às últimas inovações tecnológicas aos que se encontram em plena exclusão tecnológica; das instituições de ensino equipadas com as mais modernas tecnologias digitais aos espaços educacionais precários e com recursos mínimos para o 
exercício da função docente. O desafio maior, no entanto, ainda se encontra na própria formação profissional para enfrentar esses e tantos outros problemas" (p. 103).

Coadunamos com Marin e Penteado (2011), pois a presença das tecnologias digitais de comunicação e informação dentro do cenário educacional faz com que o professor se sinta desafiado a rever e a ampliar seus conhecimentos, provocando demandas que vão além da organização e da rotina de sala de aula, mas que também geram dificuldades, principalmente no que diz respeito ao uso dessa ou daquela tecnologia.

Gráfico 1: Dados percentuais dos artigos classificados em cada dimensão.

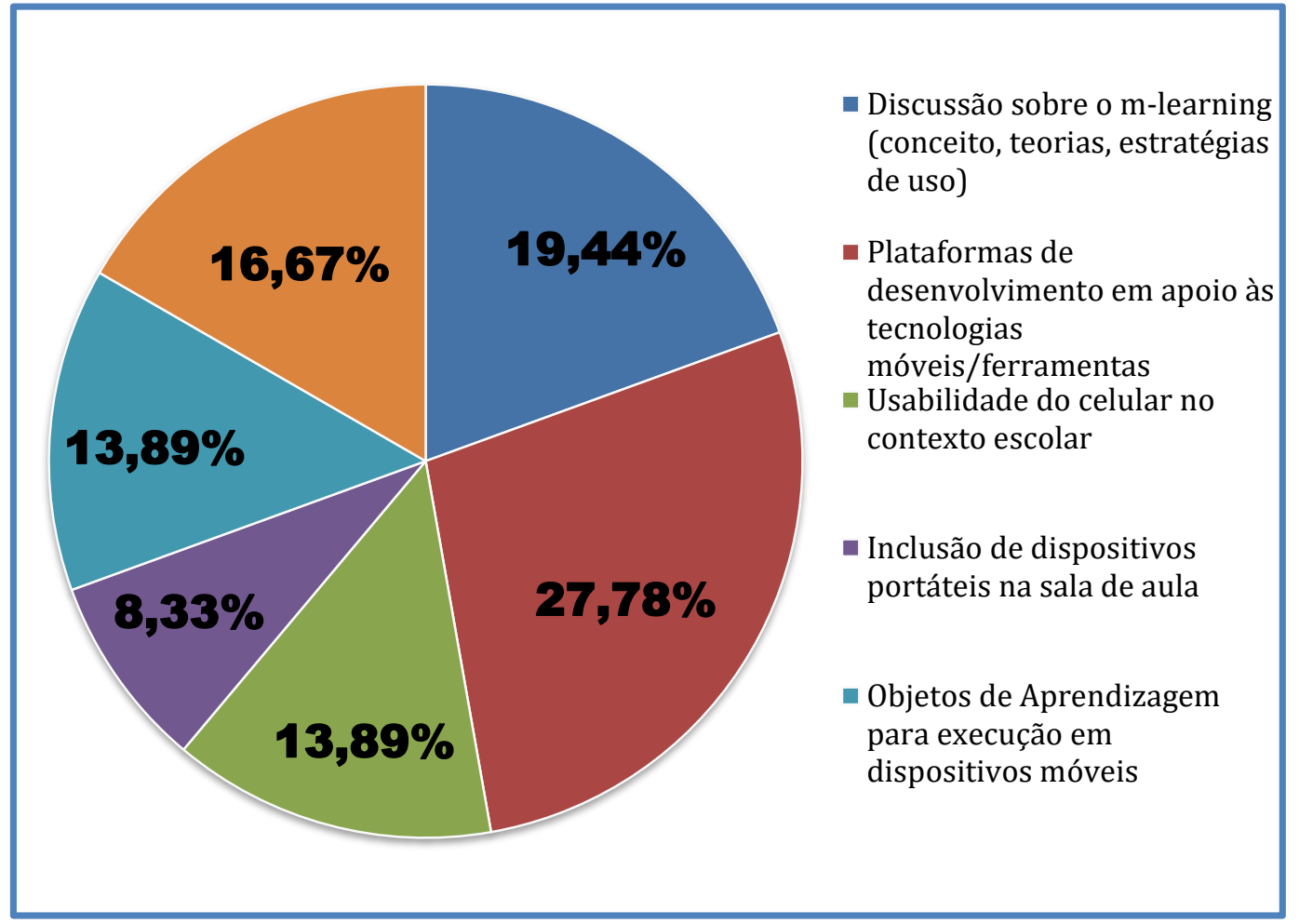

De um modo geral, pudemos estabelecer a seguinte relação decrescente entre os dados observados nas dimensões:

Plataformas de desenvolvimento em apoio às tecnologias móveis/ferramentas > Discussão sobre o m-learning (conceito, teorias, estratégias de uso) > Mobile Learning Engine Moodle (MLEMoodle) > Usabilidade do celular no contexto escolar = Objetos de Aprendizagem para execução em dispositivos móveis > Inclusão de dispositivos portáteis na sala de aula. 
Gráfico 2: Relação entre os dados observados nas dimensões.

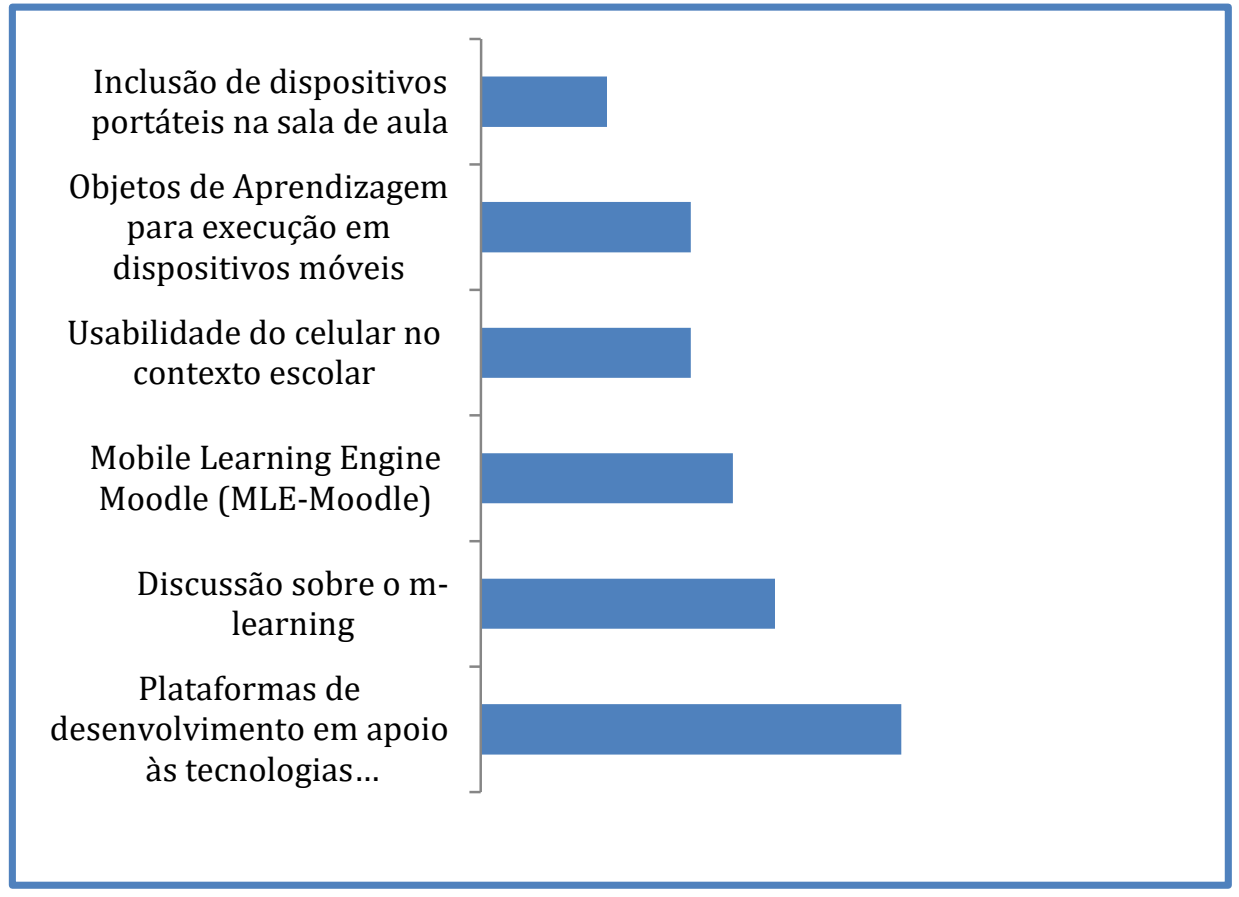

É importante frisar que um determinado artigo poderia ter sido classificado, concomitantemente, em mais de uma dimensão, a citar os artigos de Bartholo et al. (2009) e Alban et al. (2012), entre outros, cujos trabalhos foram atribuídos às dimensões "Plataformas de desenvolvimento em apoio às tecnologias moveis/ferramentas" e "Mobile Learning Engine Moodle", respectivamente, pois ambos, em sua magnitude, representam plataformas de aprendizagem. Dessa forma, o percentual referente a cada dimensão ultrapassaria os $100 \%$. Nesta pesquisa, preferimos o particionamento, e não a junção de tais dimensões.

\section{Reflexões finais}

Os resultados explanados acima foram extraídos diante do tratamento de dados obtidos a partir dos artigos analisados, os quais estavam situados dentro do espectro de palavras-chave utilizadas durante a pesquisa. Vale salientar que esta pesquisa não é estanque.

Percebemos, com esta pesquisa, que a grande preocupação, por parte dos artigos analisados sobre o m-learning, está arraigada em uma discussão "técnica", e não no saber e fazer pedagógico. Tal conclusão é extraída a partir da análise sobre as dimensões categoriais elaboradas, uma vez que, dos 36 artigos encontrados, 16 são alocados nas categorias "Plataformas de desenvolvimento em apoio às tecnologias móveis/ferramentas" e "Mobile Learning Engine Moodle", ou seja, equivalentes a 44,45\% dos artigos.

Em 2007, Saccol e Reinhard divulgaram um artigo intitulado "Tecnologias de Informação Móveis, Sem Fio e Ubíquas: Definições, Estado-da-Arte e Oportunidades de Pesquisa". Neste 
artigo, os autores apontaram algumas deficiências observadas em relação à pesquisa com mlearning. Para estes autores, a carência de definições teóricas claras e a carência de pesquisa empírica, entre outras (SACCOL e REINHARD, 2007), deixavam lacunas que poderiam tornar-se oportunidades para pesquisas futuras. $O$ que podemos observar com a nossa pesquisa é que, de 2007 para cá, os avanços em termos de pesquisas relevantes ainda podem ser considerados incipientes para o contexto brasileiro. A falta de políticas educacionais que possam estabelecer critérios de implantação sobre a legitimidade de uso dos dispositivos móveis no âmbito educacional do Brasil faz com que muitos professores não saibam fazer usufruto destes recursos, os quais apresentam índices potencialmente positivos para a educação, quando inseridos corretamente no contexto escolar.

Ainda é perceptível a dicotomia entre as diversas teorias existentes que podem dar suporte ao m-learning. $O$ fato é que avançamos pouco, e ainda existem muitas questões para serem desvendadas, em termos de sua funcionalidade e aplicação no contexto escolar. É preciso que tenhamos mudanças antológicas no tocante às concepções de alunos e professores acerca do uso do m-learning. Precisamos considerar o m-learning como sendo uma tecnologia inovadora e que pode fomentar o construtivismo social na educação, agindo como um catalisador necessário para a reforma educacional. Kukulska-Hulmw (2010) também já enfatizava a natureza positiva e catalítica do m-learning. É importante considerar também que o m-learning é um mero facilitador da aprendizagem, ou seja, um recurso pedagógico de alto potencial, no entanto, ele sozinho não garante a aprendizagem.

Não obstante, esta pesquisa nos mostra que ainda existem muitos campos para se alçar voos em relação à pesquisa com os dispositivos móveis. Para que um professor possa utilizar estes dispositivos como recurso ou ferramenta didática, basta que ele se atualize, domine alguns conhecimentos inerentes ao processo, trace estratégias metodológicas e, sobretudo, que se planeje adequadamente. No que tange à utilização do m-learning para o ensino das Ciências, percebemos, através deste estudo, que as pesquisas podem ser consideradas incipientes. Contudo, é preciso utilizar o potencial dos dispositivos móveis para praticar a inclusão digital em espaços formais, informais e não formais de educação em Ciências e em outras áreas do conhecimento, podendo, entre outras ações, promover o aprendizado de pessoas que possuam algum tipo de deficiência visual ou auditiva, etc.

\section{Agradecimentos}

À FAPEPI (Fundação de Amparo à Pesquisa do Estado do Piauí), pelo apoio na concessão de Bolsa. 


\section{Referências}

AHMED, S.; PARSONS, D. Abductive science inquiry using mobile devices in the classroom.

Computers \& Education, 63, p. 62-72, 2013.

ALBAN, A., MARCHI, A. C. B., Scortegagna, S. A.; Leguisamo, C. P. Ampliando a usabilidade de interfaces web para idosos em dispositivos móveis: uma proposta utilizando design. Revista Novas Tecnologias na Educação, 10, 3, 2012.

AUDINO, D. F.; NASCIMENTO, R. S. Objetos de aprendizagem - Diálogo entre conceitos e uma proposição aplicada à educação. Revista Contemporânea de Educação, 5, 10, 2010.

BARTHOLO, V. F., AMARAL, M. A.; CAGNIN, M. I. Uma Contribuição para a Adaptabilidade de Ambientes Virtuais de Aprendizagem para Dispositivos Móveis. Revista Brasileira de Informática na Educação, 17, 2, 2009.

BERGE, Z. L.; MUILENBURG, L. Y. Handbook of Mobile Learning. New York: Routledge, 2013.

CERTAL, F. M.; CARVALHO, A. A. Estudo sobre receptividade ao m-learning no ensino básico. VII Conferência Internacional de TIC na Educação, p. 1427-1438, 2011.

CINQUE, M.; PENSIERI, C. Campus We-Com. University students atitude towards didactical innovation. Journal of e-learning and knowledge society, 1, 57-65, 2013.

CORBEIL, J. R.; VALDES-CORBEIL, M. E. Are You Ready for Mobile Learning? In EDUCAUSE, 30, 2, 2007.

DENZIN, N. K.; LINCOLN, Y. S. Introduction: the discipline and practice of qualitative research. In: DENZIN, N. K.; LINCOLN, Y. S. (Eds.). The handbook of qualitative research. 2. ed. London: Sage, p. 1-28, 2000.

FERREIRA, J. B.; SILVA, J. F.; CAMPOS, H.; CARVALHO, M. L.; FREITAS, A. S.; SACCOL, A.; SCHLEMMER, E. A disseminação da aprendizagem com mobilidade (M-learning). Data Grama Zero - Revista de Informação, 13, 4, 2012.

NERI de SOUZA, F. Perguntas na Aprendizagem de Química no Ensino Superior. Aveiro: Universidade de Aveiro, UA, Portugal, 2006.

GRUND, F. B.; GIL, D. J. G. Mobile Learning - Los dispositivos móviles como recurso educativo. Sevilla: Eduforma, 2011.

JAMEE, K.; WONGYU, L. Meanings of criteria and norms: Analyses and comparisons of ICT literacy competencies of middle school students. Computers \& Education, 64, 81-94, 2013.

JONASSEN, D. H., CARR, C.; YUEH, H. P. Computers as Mind tools for engaging learners in critical thinking. Tech Trends, 43, 2, p.24-32, 1998. 
KITCHENHAM, B. Procedures for performing systematic reviews. Joint technical report Software Engineering Group, Keele University, United Kingdom and Empirical Software Engineering, National ICT Australia Ltd, Australia, 2004. Disponível em http://csnotes.upm.edu.my/kelasmaya/pgkm20910.nsf/0/715071a8011d4c2f482577a700386d3a LSFILE/10.1.1.122.3308\%5B1\%5D.pdf.

KUKULSA-HULME, A. Mobile learning as catalyst for change.Open Learning: The journal of open and distance learning, 25, 3, p.181-185, 2010.

LEDESMA, F. Mobile learning: Proibir ou integrar? PROFFORMA, n. 9, 2013.

LEUNG, C-H.; CHAN, Y-Y. Mobile learning: A new paradigm in electronic learning. In Proceedings of the 3rd IEEE international conference on advanced learning technologies, ed. V. Devedzic, J. Spector, D. Sampson, and Kinshuk, p.76-80. Athens, Greece: IEEE Computer Society Press, 2003. LIU, Y., HAN, S.; LI, H. Understanding the factors driving m-learning adoption: a literature review. Campus-Widelnformation Systems, 27, 4, 2010.

LÜDKE, M.; ANDRÉ, M. E. D. A. Pesquisa em educação - abordagens qualitativas. 2a ed. São Paulo: LTC (Grupo GEN), 2013.

MARIN, D.; PENTEADO, M. G. Professores que Utilizam Tecnologia de Informação e Comunicação para Ensinar Cálculo. Educação Matemática Pesquisa, 13, 3, 2011. Em MLE-Moodle. MLEMoodle. 2009. Disponível em <http://mle.sourceforge.net/mlemoodle/index.php?lang=en>. Acesso em: 10 junho. 2013.

MOURA, A. Apropriação do Telemóvel como Ferramenta de Mediação em Mobile Learning: Estudos de Caso em Contexto Educativo. Braga: Universidade do Minho, 2010.

MUYINDA, P. B. M-Learning: pedagogical, technical and organizational hypes and realities. Campus-Wide Information Systems, 24, 2, p. 97-104, 2007.

O'MALLEY, C.; VAVOULA, G.; GLEW, J.; TAYLOR, J.; SHARPLES, M.; LEFRERE, P. Guidelines for learning/teathing/tutoring in mobile environment. MOBllearn Deliverable, 4, 2003.

OKITA, S. Y, TURKAY, S., KKIN, M.; MURAI, Y. Technological design choices on learning. Computers \& Education, 63, p.176-196, 2013.

PLESCH, C., KAENDLER, C.; RUMMEL, N., WIEDMANN, M. E SPADA, H. Identifying Areas of Tension in the field of technology-enhanced learning: Results of an international Delphi study. Computers \& Education, 65, p.92-105, 2013.

PRENSKY, V. M. Digital natives, digital immigrants. On the Horizon, 9, 5, p.1-6, 2011.

KENSKI, V. M. Educação e tecnologias: o novo ritmo da informação. 5.ed. Campinas, SP: Papirus, 2009. 
RIBEIRO, P. S. E MEDINA, R. D. Mobile Learning Engine Moodle (MLE - Moodle): das funcionalidades a validação em curso a distância utilizando dispositivos móveis. Novas Tecnologias na Educação, 7, 1, 2009.

SACCOL, A. Z., SCHLEMMER, E., BARBOSA, J.; HAHN, R. M-learning e U-learning: novas perspectivas da aprendizagem móvel e ubíqua. São Paulo: Pearson Education, 2010.

SACCOL, A. Z.; REINHARD, N. Tecnologias de Informação Móveis, Sem Fio e Ubíquas: Definições, Estado-da-Arte e Oportunidades de Pesquisa. Revista de Administração Contemporânea, 11, 4, 175-198, 2007.

SCORNAVACCA, E., HUFF, S.; MARSHALL, S. Mobile phones in the classroom: if you can't beat them, join them. Communications of the ACM, 52, 4, p.142-146, 2009.

SHARPLES, M. Big issues in mobile learning. Report of a workshop by the Kaleidoscope Network of Excellence Mobile Learning Initiative. UK: University of Nottingham, 2006.

SOSTERIC, M.; HESEMEIER, S. When is a Learning Object not na Objetct. A first step towards a theory of learning objects. Internacional Review of Research in Open and Distance Learning, 3, 2, 2002.

TRAXLER, J. Defining, Discussing and Evaluating Mobile Learning: the moving finger writes and having writ. The International Review of Research in Open and Distance Learning, 8, 2, 2007. TRAXLER, J. Current State of Mobile Learning. Mobile Learning: Transforming the Delivery of Education and Training, p. 9-24. AU Press, Athabasca University, 2009.

TRAXLER, J. Introduction. In J. Traxler.; J. Wishat (Eds.), Making mobile learning work: case estudies of practice, p. 4-12. Bristol, UK: Escalate Education Subject Centre: advanced learning and teaching in education, 2013.

TWISS, T. Ubiquitous information: An eFellow report on the use of mobile phones in classrooms to foster information literacy, 2008. Disponível em http://www.scribd.com/doc/9507014/ToniTwiss-Ubiquitous-Information/.

WANG, Y-K. Context Awareness and Adaptation in Mobile Learning. 2nd International Workshop on Wireless and Mobile Technologies in Education, p.154-158, 2004.

WILEY, D. Connecting learning objects to instructional design theory: a definition, a metaphor, and a taxonomy. The Instructional Use of Learning Objects, [S.I.], p.571-577, 2002.

WONG, L. H.; LOOI, C. K. What seams do we remove in mobile assisted seamless learning? A critical review of the literature. Computers \& Education, 57, 4, p.2364-2381, 2011. 


\section{Anexo 1}

Tabela: Categoria, revistas, títulos dos artigos e dados de divulgação.

\begin{tabular}{|c|c|c|c|}
\hline DIMENSÃO & REVISTA & TíTULO & $\begin{array}{l}\text { DADOS DE } \\
\text { DIVULGAÇÃO }\end{array}$ \\
\hline a) & $\begin{array}{l}\text { Revista de } \text { gestão } \mathrm{da} \\
\text { tecnologia e } \\
\text { informação }\end{array}$ & $\begin{array}{l}\text { M-LEARNING (MOBILE LEARNING) IN } \\
\text { PRACTICE: A TRAINING EXPERIENCE WITH } \\
\text { IT PROFESSIONALS }\end{array}$ & Vol.7, №2, 2010 \\
\hline a) & $\begin{array}{l}\text { Novas tecnologias na } \\
\text { educação }\end{array}$ & $\begin{array}{lrr}\text { APRENDIZAGEM COM } & \text { MOBILIDADE NA } \\
\text { PERSPECTIVA DIALÓGICA: } & \text { REFLEXÕES E } \\
\text { POSSIBILIDADES } & \text { PARA } & \text { PRÁTICAS } \\
\text { PEDAGÓGICAS } & & \\
\end{array}$ & Vol.7, №3, 2009. \\
\hline a) & $\begin{array}{l}\text { Novas tecnologias na } \\
\text { educação }\end{array}$ & $\begin{array}{l}\text { CONTRIBUIÇÕES DA TEORIA DA ATIVIDADE } \\
\text { PARA M-LEARNING }\end{array}$ & Vol.8, №2, 2010. \\
\hline a) & $\begin{array}{l}\text { Novas tecnologias } \\
\text { educação }\end{array}$ & $\begin{array}{l}\text { UBIQUIDADE E MOBILIDADE DE OBJETOS } \\
\text { DE APRENDIZAGEM USANDO O PAPEL } \\
\text { COMO RECURSO }\end{array}$ & Vol.8, №3, 2010 \\
\hline a) & $\begin{array}{l}\text { Novas tecnologias } \\
\text { educação }\end{array}$ & $\begin{array}{l}\text { M-LEARNING E ANDROID: UM NOVO } \\
\text { PARADIGMA? }\end{array}$ & $\begin{array}{l}\text { Vol.6, } \\
\text { №2, } 2008 .\end{array}$ \\
\hline a) & Revista educação online & $\begin{array}{llrr}\text { DO } & \text { COMPUTADOR } & \text { AO } & \text { TABLET: } \\
\text { VANTAGENS } & \text { PEDAGÓGICAS } & \text { NA } \\
\text { UTILIZAÇÃO DE } & \text { DISPOSITIVOS MÓVEIS NA } \\
\text { EDUCAÇÃO } & & \end{array}$ & $\begin{aligned} \text { Vol.6, } \\
\text { №1, } 2012 .\end{aligned}$ \\
\hline a) & Udescvirtu@I - online & $\begin{array}{lccc}\text { DO } & \text { E-LEARNING AO M-LEARNING - } \\
\text { REFLEXÃO PARA A MUDANÇA }\end{array}$ & Vol.2, №2, 2009. \\
\hline b) & $\begin{array}{l}\text { Novas tecnologias } \\
\text { educação }\end{array}$ & $\begin{array}{l}\text { DESENVOLVIMENTO DE APLICAÇÃO PARA } \\
\text { INTERFACEAMENTO COM EXPERIMENTOS } \\
\text { REMOTOS POR SMARTHPHONES. }\end{array}$ & $\begin{array}{l}\text { Vol.10, } \\
2012 .\end{array}$ \\
\hline b) & $\begin{array}{l}\text { Novas tecnologias } \\
\text { educação }\end{array}$ & $\begin{array}{l}\text { ANÁLISE DAS PLATAFORMAS } \\
\text { DESENVOLVIMENTO MOBILE APLICADOS } \\
\text { NA ÁREA EDUCACIONAL, USANDO } \\
\text { ANDROID E WINDOWS PHONE. ESTUDO } \\
\text { DE CASO: APLICATIVO PLANETAS NO } \\
\text { WINDOWS PHONE. }\end{array}$ & $\begin{array}{l}\text { Vol.10, } \\
2012 .\end{array}$ \\
\hline b) & $\begin{array}{l}\text { Novas tecnologias } \\
\text { educação }\end{array}$ & $\begin{array}{l}\text { DESENVOLVIMENTO DE APLICAÇÕES M- } \\
\text { LEARNING NAS PLATAFORMAS J2ME E } \\
\text { FLASH LITE. }\end{array}$ & Vol.5, №2, 2007. \\
\hline b) & $\begin{array}{l}\text { Novas tecnologias } \\
\text { educação }\end{array}$ & $\begin{array}{l}\text { EM DIREÇÃO A EDUCAÇÃO UBÍQUA: } \\
\text { APRENDER SEMPRE, EM QUALQUER } \\
\text { LUGAR, COM QUALQUER DISPOSITIVO. }\end{array}$ & Vol.6, №2, 2008. \\
\hline b) & $\begin{array}{l}\text { Novas tecnologias } \\
\text { educação }\end{array}$ & \begin{tabular}{lll}
\multicolumn{3}{l}{ AMPLIANDO AS FUNCIONALIDADES DE UM } \\
SISTEMA & GERENCIADOR & DE \\
APRENDIZAGEM & PARA O O CONTEXTO & DA \\
TELEMÁTICA MÓVEL. & & \\
\end{tabular} & Vol.6, №1, 2008. \\
\hline b) & Informática na educação & $\begin{array}{l}\text { APERFEIÇOAMENTO AUTOMÁTICO } \\
\text { PERFIL DO APRENDIZ EM AMBIENTES DE } \\
\text { EDUCAÇÃO UBÍQUA }\end{array}$ & $\begin{array}{l}\text { Vol.16, } \\
2008 .\end{array}$ \\
\hline b) & Informática na educação & $\begin{array}{l}\text { UMA CONTRIBUIÇÃO } \\
\text { ADAPTABILIDADE DE AMBIENTES VIRTUAIS } \\
\text { DE APRENDIZAGEM PARA } \\
\text { DISPOSITIVOS } \\
\text { MÓVEIS }\end{array}$ & $\begin{array}{l}\text { Vol.17, } \\
2009 .\end{array}$ \\
\hline b) & $\begin{array}{l}\text { Informática na educação: } \\
\text { teoria \& prática }\end{array}$ & $\begin{array}{l}\text { MATERIALES DE APRENDIZAJE MÓVIL EN } \\
\text { CONTEXTOS CRÍTICOS }\end{array}$ & $\begin{array}{l}\text { Vol.14, } \\
2011 .\end{array}$ \\
\hline b) & Informática na educação: & $\begin{array}{lll}\text { APERFEIÇOAMENTO } & \text { AUTOMÁTICO } & \text { DO }\end{array}$ & Vol.16, \\
\hline
\end{tabular}

DOI: Em andamento. 


\begin{tabular}{|c|c|c|c|}
\hline & teoria \& prática & $\begin{array}{l}\text { PERFIL DO APRENDIZ EM AMBIENTES DE } \\
\text { EDUCAÇÃO UBÍQUA. }\end{array}$ & 2008. \\
\hline c) & $\begin{array}{l}\text { Informática na educação: } \\
\text { teoria \& prática }\end{array}$ & $\begin{array}{l}\text { UMA CONTRIBUIÇÃO } \\
\text { ADAPTABILIDADE DE AMBIENTES VIRTUAIS } \\
\text { DE APRENDIZAGEM } \\
\text { MÓVEIS. }\end{array}$ & $\begin{array}{l}\text { Vol.17, } \quad \text { №2, } \\
2009 .\end{array}$ \\
\hline b) & $\begin{array}{l}\text { Educação, formação e } \\
\text { tecnologia }\end{array}$ & $\begin{array}{l}\text { PEDDY-PAPER LITERÁRIO MEDIADO POR } \\
\text { TELEMÓVEL. }\end{array}$ & Vol.2, №2, 2009. \\
\hline c) & $\begin{array}{l}\text { Educação, formação e } \\
\text { tecnologia }\end{array}$ & JOVENS, TELEMÓVEIS E ESCOLA. & № extra, 2010. \\
\hline b) & $\begin{array}{l}\text { Novas tecnologias na } \\
\text { educação }\end{array}$ & $\begin{array}{l}\text { M-LEARNING E MATEMÁTICA: MAPEANDO } \\
\text { RECURSOS E } \\
\text { EDUCACIONAIS. }\end{array}$ & Vol.7, №3, 2009. \\
\hline c) & Revista educaonline & $\begin{array}{l}\text { A ADOPÇÃO E DIFUSÃO DE PRÁTICAS DE } \\
\text { M-LEARNING NO CONTEXTO DO ENSINO } \\
\text { SUPERIOR: UM ESTUDO DE AVALIAÇÃO DO } \\
\text { USO DE DISPOSITIVOS MÓVEIS EM } \\
\text { AMBIENTES COLABORATIVOS. }\end{array}$ & Vol.5, №2, 2011. \\
\hline c) & Revista educação online & $\begin{array}{llllr}\text { TECNOLOGIAS MÓVEIS: O } & \text { USO } & \text { DO } \\
\text { CELULAR COMO SUPORTE } & \text { PARA } & \text { A } \\
\text { EDUCAÇÃO. } & & & & \\
\end{array}$ & Vol.7, №1, 2013. \\
\hline d) & $\begin{array}{l}\text { Educação, formação } \mathrm{e} \\
\text { tecnologia }\end{array}$ & $\begin{array}{l}\text { PORTÁTEIS NA SALA DE AULA - PROJECTO } \\
\text { NAVEGAR: UMA JANELA COM VISTAS } \\
\text { PARA A FRENTE. }\end{array}$ & Vol.1, №1, 2008. \\
\hline d) & $\begin{array}{l}\text { Educação, formação e } \\
\text { tecnologia }\end{array}$ & $\begin{array}{l}\text { O COMPUTADOR PORTÁTIL NO AMBIENTE } \\
\text { DE SALA DE AULA NUMA ESCOLA DO } \\
\text { ALENTEJO LITORAL. }\end{array}$ & Vol.3, №1, 2010. \\
\hline d) & Acta scientiarum. education & $\begin{array}{lrrr}\text { STUDENT } & \text { PERSPECTIVES } & \text { ABOUT } & \text { MOBILE } \\
\text { LEARNING INITIATIVES } & \text { AT } & \text { OPEN } \\
\text { UNIVERSITY OF } & \text { BRAZIL: THE } & \text { MOBILE } \\
\text { PHONE ISSUE. } & & & \\
\end{array}$ & $\begin{array}{l}\text { Vol.32, №2, } \\
2010 .\end{array}$ \\
\hline e) & $\begin{array}{l}\text { Novas tecnologias na } \\
\text { educação }\end{array}$ & $\begin{array}{l}\text { MOBILE LEARNING NA SALA DE AULA } \\
\text { PRESENCIAL: REPOSITÓRIO SEMÂNTICO DE } \\
\text { OBJETOS DE APRENDIZAGEM PARA } \\
\text { DISPOSITIVOS MÓVEIS. }\end{array}$ & Vol.7, №3, 2009. \\
\hline e) & $\begin{array}{l}\text { Novas tecnologias na } \\
\text { educação }\end{array}$ & $\begin{array}{lccr}\text { SISTEMA } & \text { DE } & \text { GERENCIAMENTO } & \text { DE } \\
\text { OBJETOS } & \text { DE } & \text { APRENDIZAGEM } & \text { PARA } \\
\text { DISPOSITIVOS MÓVEIS. }\end{array}$ & Vol.7, №1, 2009. \\
\hline e) & $\begin{array}{l}\text { Novas tecnologias na } \\
\text { educação }\end{array}$ & $\begin{array}{l}\text { DESENVOLVIMENTO DE OBJETOS DE } \\
\text { APRENDIZAGEM PARA DISPOSITIVOS } \\
\text { MÓVEIS: UMA NOVA ABORDAGEM QUE } \\
\text { CONTRIBUI PARA A EDUCAÇÃO. }\end{array}$ & - \\
\hline e) & Informática na educação & $\begin{array}{l}\text { M-TUTORIAL: FERRAMENTA DE AUTORIA } \\
\text { PARA DESENVOLVIMENTO DE TUTORIAIS } \\
\text { VOLTADOS PARA O M-LEARNING. }\end{array}$ & $\begin{array}{l}\text { Vol.20, } \\
2012 .\end{array}$ \\
\hline e) & $\begin{array}{l}\text { Informática na educação: } \\
\text { teoria \& prática }\end{array}$ & $\begin{array}{l}\text { COMPUTAÇÃO MÓVEL E UBÍQUA NO } \\
\text { CONTEXTO DE UMA GRADUAÇÃO DE } \\
\text { REFERÊNCIA. }\end{array}$ & $\begin{array}{l}\text { Vol.15, №3, } \\
2007 .\end{array}$ \\
\hline f) & $\begin{array}{l}\text { Novas tecnologias } \\
\text { educação }\end{array}$ & $\begin{array}{l}\text { MODELAGEM DE UM FRAMEWORK PARA } \\
\text { ADAPTAÇÃO DE AMBIENTES VIRTUAIS DE } \\
\text { APRENDIZAGEM MÓVEIS AOS DIFERENTES } \\
\text { ESTILOS COGNITIVOS. }\end{array}$ & Vol.7, 2009. \\
\hline f) & $\begin{array}{l}\text { Novas tecnologias na } \\
\text { educação }\end{array}$ & $\begin{array}{l}\text { MOBILE LEARNING ENGINE MOODLE (MLE } \\
\text { - MOODLE): DAS FUNCIONALIDADES A }\end{array}$ & Vol.7, №1, 2009. \\
\hline
\end{tabular}




\begin{tabular}{|c|c|c|c|}
\hline & & $\begin{array}{l}\text { VALIDAÇÃO EM CURSO A DISTÂNCIA } \\
\text { UTILIZANDO DISPOSITIVOS MÓVEIS. }\end{array}$ & \\
\hline f) & $\begin{array}{l}\text { Novas tecnologias na } \\
\text { educação }\end{array}$ & $\begin{array}{l}\text { MOBILE LEARNING ENGINE } \text { MOODLE } \\
\text { ADAPTADO AOS DIFERENTES ESTILOS } \\
\text { COGNITIVOS UTILIZANDO HIPERMIIDIA } \\
\text { ADAPTATIVA. }\end{array}$ & Vol.8, №2, 2010. \\
\hline f) & $\begin{array}{l}\text { Novas tecnologias na } \\
\text { educação }\end{array}$ & $\begin{array}{l}\text { CELULAR COMO FERRAMENTA DE APOIO } \\
\text { PEDAGÓGICO AO CÁLCULO. }\end{array}$ & Vol.9, №1, 2011. \\
\hline f) & $\begin{array}{l}\text { Novas tecnologias } \\
\text { educação }\end{array}$ & $\begin{array}{lccr}\text { AMPLIANDO } & \text { A } & \text { USABILIDADE } & \text { DE } \\
\text { INTERFACES } & \text { WEB PARA IDOSOS } & \text { EM } \\
\text { DISPOSITIVOS } & \text { MÓVEIS: UMA PROPOSTA } \\
\text { UTILIZANDO DESIGN RESPONSIVO. }\end{array}$ & $\begin{array}{l}\text { Vol.10, } \\
2012 .\end{array}$ \\
\hline f) & $\begin{array}{l}\text { Novas tecnologias na } \\
\text { educação }\end{array}$ & $\begin{array}{l}\text { APRENDIZAGEM COM MOBILIDADE PARA } \\
\text { AS ATIVIDADES DE PRÁTICA EM CURSOS } \\
\text { DE LICENCIATURA. }\end{array}$ & Vol.4, №1, 2007. \\
\hline
\end{tabular}

Maria das Graças Cleophas, é Doutora em Ensino das Ciências (Ênfase: Ensino de Química) e Professora Adjunta do Instituto Latino-Americano da Ciências da Vida e da Natureza (ILACVN) da Universidade Federal da Integração Latino-Americana (UNILA), Foz do Iguaçu - PR. E-mail: mgcp76@gmail.com

Eduardo Luiz Dias Cavalcanti, é professor da Universidade de Brasília (UNB) na Divisão de Ensino de Química do Instituto de Química IQ-UnB. E-mail: eldcquimica@yahoo.com.br

Francislê Neri de Souza tem pós-doutoramento em Tecnologias da Informação e Comunicação (TIC) aplicados ao Ensino de Ciências e atualmente trabalha como professor na Universidade de Aveiro-Portugal.E-mail: $\underline{\text { fns@ua.pt }}$

Marcelo Brito Carneiro Leão, é Pós-Doutor no uso das Tecnologias da Informação e Comunicação no Ensino de Ciências pela Universitat de Barcelona (2006), professor associado da Universidade Federal Rural de Pernambuco (UFRPE), onde é Bolsista de Produtividade DT do CNPq e Coordenador do Núcleo SEMENTE (Sistemas para a Elaboração de Materiais Educacionais com uso de Novas Tecnologias) da UFRPE. E-mail: $\underline{\text { mbcleao@terra.com.br }}$

DOI: Em andamento. 